\title{
Conceptions des agriculteurs et modèles agronomiques. Le pâturage des vaches laitières dans le Jura
}

\author{
Anne Mathieu \\ Agronome, INRA SAD-APT, BP 01, 78850 Thiverval-Grignon, France
}

L'adaptation des pratiques en fonction des aléas climatiques n'est pas une question anodine dans le contexte actuel et futur. Les mots des éleveurs, au rythme des saisons, nous font entrer dans les formes de connaissance mobilisées dans la conduite du pâturage. Les acteurs locaux ont en effet une production de connaissances qui rend compte de leur expérience de la complexité. Le statut de ces savoirs locaux dans la production scientifique reflète des postures disciplinaires particulières : celle du socioanthropologue qui les révèle, et celle de l'agronome qui les utilise pour améliorer ses modèles. L'enjeu interdisciplinaire est bien là, dans la nécessité d'une mise à l'épreuve de ces points de vue, ceux des éleveurs comme ceux des scientifiques, chacun pouvant avoir une pertinence, mais étant nécessairement limité.

La Rédaction

\begin{abstract}
Mots-clés :
Résumé - Les agriculteurs développent des connaissances des processus techniques utiles pour l'action. conduite du pâturage; Ce ne sont pas toujours les mêmes que celles des agronomes. Pour la conduite du pâturage des vaches pratiques ; conceptions d'agriculteurs ; plateau du Jura laitières, nous avons mis en évidence des conceptions d'agriculteurs du Jura, à partir d'une méthode d'analyse de leurs discours et d'observations de leurs pratiques. Ces conceptions et ces pratiques sont confrontées à la façon dont les agronomes conçoivent le pâturage tournant. Les façons de concevoir les relations entre l'espace et le temps sont différentes dans les deux cas. Les agriculteurs enquêtés considèrent l'évolution de l'herbe dans des endroits différents à chaque moment ; pour les agronomes, les parcelles sont des espaces fixes pendant toute la saison, et l'herbe évolue au même endroit quand le temps s'écoule. La surface enjeu de la conduite du pâturage n'est pas la même pour ces deux groupes. Pour les agriculteurs, l'enjeu est la surface des pâtures en juillet, considérée comme une conséquence de ce qui s'est passé au printemps. Dans le pâturage tournant, c'est la surface au printemps, déterminée avant même la sortie des vaches, qui est l'enjeu de la conduite. L'auteur montre aussi que les indicateurs de conduite ne sont pas les mêmes dans les deux cas. Une discussion est réalisée sur les possibilités d'utilisation des connaissances sur les conceptions des agriculteurs par les agronomes, et sur les généralisations des résultats présentés.
\end{abstract}

\section{Keywords:}

grazing management; practices; farmer conceptions; Jura Mountains

\begin{abstract}
Comparing farmer conceptions with scientific models. Grazing management for dairy cows in the Jura Mountains. Farmers develop knowledge on technical processes that is useful for action. As such their knowledge often differs from that of agricultural scientists. A study on pasture management for dairy cows in the Jura Mountains enabled the author to highlight the farmers' views by analyzing their discourse and observing their practices. Their conceptions and practices were then compared with the agricultural scientists' conception of rotational grazing. Farmers and agricultural scientists conceive differently the relations between space and time. The farmers interviewed follow the growth of grass at different sites at each moment in time whereas agricultural scientists view a pasture as an area of land that is fixed throughout the grazing season, with grass growing on that field with the passage of time. The issue of the grazing area in grazing management differs in the two groups. For the Jura farmers, the main issue is the size of the grazing area in July, which is considered to be a result of what has occurred in the spring. In rotational grazing management, the chief consideration is the grazing area available in the spring, which is determined before the cows are turned out to grass. Management indicators are also shown to differ in the two situations. Potential use by agricultural scientists of their knowledge of farmer conceptions, and the transferability of the results are presented are discussed.
\end{abstract}

Auteur correspondant : amathieu@grignon.inra.fr 


\section{Introduction}

Depuis longtemps, les chercheurs du département Systèmes agraires et développement de l'Inra cherchent à comprendre et à modéliser les pratiques des agriculteurs (Landais et Deffontaines, 1990), soit pour en proposer des améliorations ou des transformations, soit pour construire des outils d'aide à la décision. Les démarches utilisées jusqu'à présent par les agronomes s'appuient sur des théories agronomiques concernant les systèmes de culture, et intègrent les théories de la gestion de l'entreprise ou de l'intelligence artificielle (Papy, 1994 ; Girard, 1995; Aubry, 2000). Les pratiques sont enregistrées, des régularités sont repérées, et elles sont interprétées à partir de connaissances biotechniques ou formalisées sous forme de règles de décision. Ces modèles sont donc une représentation de ce que l'agriculteur fait, traduit par des agronomes.

Cependant, les conceptions que les agriculteurs ont d'un processus technique peuvent être notablement différentes de celles des agronomes. En effet, ces deux groupes d'acteurs n'ont pas la même activité, ce qui leur confère un point de vue différent sur le processus technique. En linguistique, Prieto (1975) montre qu'en conséquence, leurs façons de découper la réalité en catégories, d'y associer des mots et de les qualifier par certains traits, sont différentes. En anthropologie, plus exactement en ethnoscience, Friedberg (1997) montre que le découpage en entités discrètes dans l'ensemble des plantes, et la façon de leur attribuer des termes, peut se faire de façon différente selon qu'il s'agit de savoirs de botanistes ou de savoirs de sociétés locales dans leurs rapports avec leur environnement. Dans ce dernier cas, l'auteur constate que l'existence des discontinuités est liée à des fonctions spécifiques de certaines plantes dans la société, ces fonctions pouvant être matérielles, alors liées à des usages ou à des repères écologiques, ou symboliques pour représenter l'ordre du monde. Dans la perspective dans laquelle nous nous situons, l'analyse des différentes conceptions d'un processus technique permet de comprendre en quoi certaines prescriptions proposées par les agronomes peuvent être difficiles à utiliser par des agriculteurs (Darré, 1985; Friedberg et al., 2000). Cela peut déboucher sur des propositions pour adapter les recommandations techniques.

L'étude présentée ici a pour origine une recherche menée en 1995 sur les adaptations aux aléas climatiques faites par des agriculteurs du deuxième plateau du Jura au cours de la conduite du pâturage. Les outils agronomiques utilisés pour enregistrer les pratiques, à savoir des calendriers d'utilisation des surfaces fourragères et des propositions de différents scénarios climatiques, ne permettaient pas un dialogue satisfaisant avec les agriculteurs, ni la formalisation de règles de décision. J'ai donc analysé les conceptions d'un groupe de ces éleveurs ${ }^{1}$. Les résultats comprennent deux parties : d'abord, je rappelle ce qu'est la conception commune à l'ensemble de ces éleveurs, quelle que soit leur façon de conduire le pâturage. Ces travaux sont détaillés par ailleurs (Mathieu, 2004). Ensuite, je montre en quoi certains points de ces conceptions rendent difficile la compréhension des modèles agronomiques pour ces éleveurs.

Remarquons que ce travail se situe à un niveau particulier, qui est celui de la conduite au jour le jour du pâturage, c'est-à-dire au niveau de ce que les agronomes appellent le pilotage, et non à celui de la définition de stratégies que je n'aborde pas ici.

\section{Présentation de la région d'étude et des enjeux du pâturage}

La zone d'étude se situe sur le deuxième plateau du Jura, à une altitude comprise entre 800 et $900 \mathrm{~m}$. C'est une zone plate de sols calcaires peu profonds. Elle est très arrosée, même pendant l'été ; sauf pour de très courtes périodes, il n'y a pas de sécheresse. C'est surtout la température qui limite la croissance des prairies. La production agricole provient presque exclusivement de l'élevage laitier, avec une transformation du lait en fromage à pâte pressée cuite (le Comté, principalement). La production de viande est un sous-produit de l'élevage laitier.

L'alimentation hivernale des animaux est à base de foin et de regain. L'ensilage est interdit à cause de la transformation du lait en fromage de Comté. La saison de pâturage dure d'avril à octobre. Le pâturage individuel des troupeaux n'existe dans cette zone que depuis les années 1970; auparavant, tous les troupeaux du village étaient rassemblés pour le pâturage. Dans la plupart des cas, le pâturage est conduit avec une clôture électrique qui permet de délimiter ce qui est donné chaque jour à pâturer aux vaches. En juin, l'herbe qui n'a pas déjà été pâturée épie, puis durcit, et elle n'est alors plus consommable au pâturage. Elle sera fauchée.

La consommation de l'herbe au pâturage se fait au fur à mesure de sa croissance : il y a peu de reports $\mathrm{d}^{\prime}$ herbe sur pied ${ }^{2}$. Il n'y a pas vraiment de concurrence entre pâturage et foin : l'alimentation au pâturage est prioritaire; dans le cas où il n'y a pas assez de foin, il y a

\footnotetext{
${ }^{1}$ La recherche sur les conceptions des éleveurs a été entreprise dans le cadre d'un séminaire de recherche, intitulé « Maîtrise et moyens d'analyse du dialogue chercheurs-techniciens ", animé par J.-P. Darré, socio-anthropologue, et financé par le département de recherche Systèmes agraires et développement de l'Inra entre 1997 et 1999.

${ }^{2}$ Ce qui est nommé reports d'herbe sur pied par les agronomes est constitué d'herbe qu'on laisse vieillir dans certaines surfaces de l'exploitation pour être consommée en pâturage après sa pousse. Cette herbe n'est pas de très bonne qualité, mais permet une réserve alimentaire.
} 
achat. Cependant, l'agrandissement des exploitations et le climat des années précédentes font qu'au moment de l'enquête, presque toutes les exploitations possèdent des excédents de foin.

\section{Matériel et méthode}

\section{La conduite des enquêtes}

L'enquête a pour objet à la fois les pratiques des éleveurs et leurs conceptions. Elle a été faite en deux parties :

- un entretien individuel, au cours duquel l'enquêteur propose à l'interviewé de parler librement de la question technique qu'il étudie. À partir d'une consigne initiale, l'interviewé est laissé libre sur les orientations du contenu. Il est incité à dire ce qui d'ordinaire est évident et va sans dire. L'enquêteur l'aide à explorer sa pensée sur le sujet. Les entretiens sont enregistrés et retranscrits intégralement. Dans cette étude, la consigne de départ de l'entretien a été : «Pouvez-vous me dire comment vous faites à la belle saison? »

- une enquête sur les caractéristiques de l'exploitation, les pratiques des agriculteurs concernant la conduite des troupeaux et des surfaces fourragères, et les résultats de l'exploitation.

\section{L'analyse des entretiens}

La possibilité de mise en évidence d'un système de pensée par une analyse de la parole réside dans l'équivalence entre la façon de concevoir les choses, c'est-àdire le sens $q u^{\prime}$ on donne aux choses, et le sens qu'on donne aux mots. Le moyen essentiel pour mettre en lumière le sens des mots est une analyse contextuelle, issue de méthodes linguistiques (Prieto, 1975). Il s'agit d'analyser les contextes dans lesquels l'interviewé utilise un mot, autrement dit à quels autres mots de la phrase ce mot est opposé, associé, ou considéré comme semblable. Cette analyse contextuelle est réalisée entretien par entretien, puis une synthèse est faite sur l'ensemble des entretiens.

Au préalable, les thèmes abordés dans le discours sont repérés. L'analyse se fait à partir des oppositions centrales que l'agriculteur opère.

La synthèse des analyses des différents entretiens peut être axée plus sur le fond commun, c'est-à-dire la façon commune de tous les éleveurs de parler de la conduite, quelles que soient leurs pratiques, ou plus sur les variantes, c'est-à-dire ce qui justifie les différentes façons de faire. C'est le fond commun de la conception de la conduite du pâturage que je rapporte dans l'étude présentée ici.

\section{Le choix de l'échantillon}

Pour avoir accès aux conceptions locales, j'ai enquêté des agriculteurs d'une même commune. Dans cette zone à habitat groupé, ils connaissent bien ce que font les uns et les autres (Moisan, 1988), et ils sont soumis à des enjeux communaux communs. La commune enquêtée a été choisie pour trois raisons :

- elle appartient à une zone dans laquelle j'avais fait des enquêtes sur la conduite de pâturages (Mathieu et Fiorelli, 1990);

- les conduites de pâturage présentes dans cette commune sont variées ;

- son altitude élevée dans la zone choisie rend l'adaptation de la conduite de printemps aux variations du climat plus difficile, en raccourcissant la période entre la sortie des animaux et la montaison des graminées. Dans une telle situation relativement tendue, les éleveurs sont mieux à même d'expliciter leurs modes de conduite que dans des conditions plus «faciles », car ils sont tenus de les raisonner plus finement.

Les quatre agriculteurs de la commune ont été enquêtés. J'ai de plus utilisé un entretien réalisé auprès d'un éleveur âgé d'une commune voisine qui utilise un type de conduite de pâturage qui n'est pas présent dans la commune étudiée.

\section{Les caractéristiques des exploitations enquêtées}

Les exploitations enquêtées sont toutes en régime de croisière, faisant fonctionner leur pâturage depuis plusieurs années. Les caractéristiques succinctes des exploitations du village sont les suivantes. Deux groupes apparaissent : deux exploitations familiales, avec des bâtiments anciens aménagés, des productions par vache entre 5500 et $6000 \mathrm{~kg}$ de lait par an; et deux $\mathrm{Gaec}^{3}$ formés par le regroupement de deux fois deux exploitations du village quelques années avant l'enquête, avec des bâtiments neufs, des quotas laitiers et des productions par vache plus élevées (plus de $7000 \mathrm{~kg}$ de lait par an). Les exploitations sont présentées dans l'Encadré, et identifiées par le prénom (modifié) de la personne interviewée.

\section{Résultats sur la conception de la conduite du pâturage}

Rappelons que nous étudions les conceptions au niveau de la conduite du pâturage au jour le jour.

\footnotetext{
${ }^{3}$ Gaec: Groupement agricole d'exploitation en commun. Statut juridique d'exploitation qui permet la présence de plusieurs chefs d'exploitation, associés.
} 


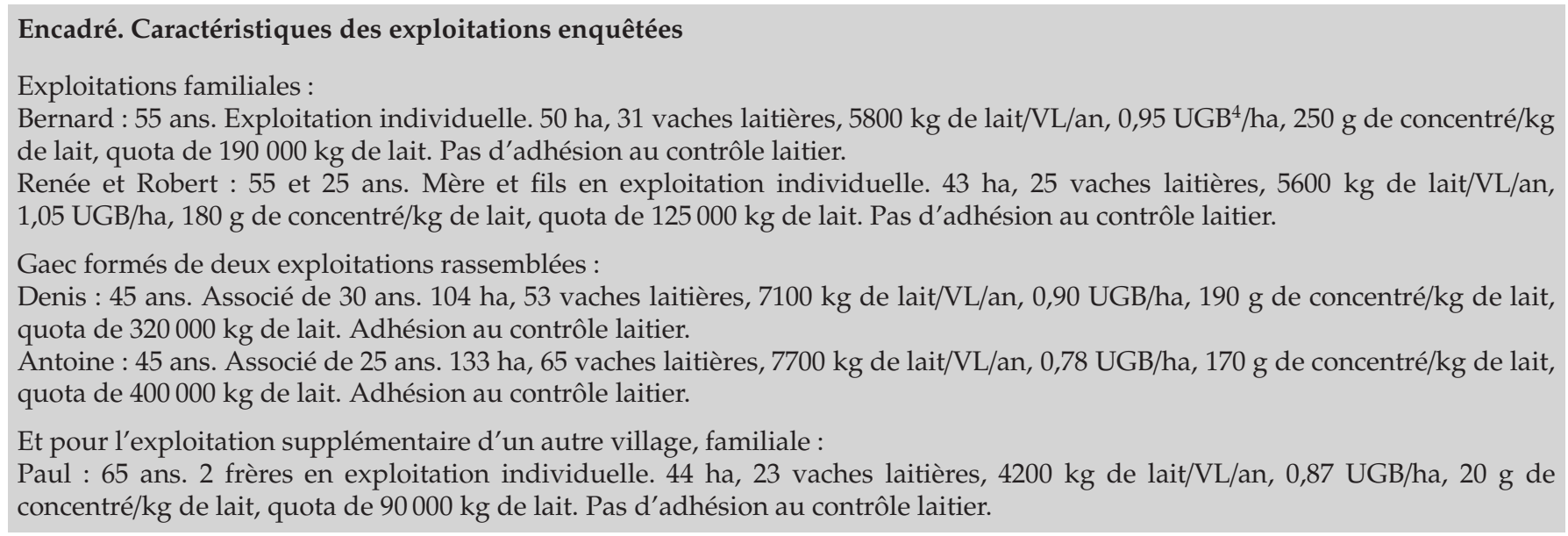

Les éleveurs enquêtés distinguent les vaches (en lactation), les taries et les génisses.

\section{La conception de l'alimentation au pâturage et de la production}

\section{Au pâturage, les éleveurs recherchent avant tout une régularité de l'approvisionnement en herbe}

Jamais le niveau de production des vaches laitières n'est évoqué dans les entretiens. Ce que recherchent les éleveurs, c'est «qu'elles ne baissent pas au lait ${ }^{5}$ ». Cette baisse entraîne une perte, mais celle-ci est généralement considérée comme de peu d'importance. Surtout, elle s'accompagne de la peur que la production ne remonte pas et soit un signe de dysfonctionnement du troupeau, qui pourrait durer et se traduire par des problèmes de fécondité : «Si tu te bases sur la production de lait, encore, tu y arrives. Mais il faut voir tout, l'état d'engraissement des vaches, et puis les autres problèmes » (Antoine). À cet objectif de production régulière, les éleveurs associent une crainte de «manquer d'herbe dans les pâtures". Des compensations avec d'autres types d'aliments ne suffisent pas. Au cours des entretiens est sans cesse évoquée la peur d'« être pris, bloqué, coincé », parce qu'il n'y a plus suffisamment $\mathrm{d}^{\prime}$ herbe à pâturer.

On retrouve un résultat déjà mis en évidence par Darré et al. (1993) dans un élevage ovin. Pour l'éleveur en question, le but central n'est pas exprimé comme un niveau de production à atteindre, mais porte sur un bon fonctionnement du troupeau et de l'ensemble du système.

\footnotetext{
${ }^{4}$ UGB : unité gros bovin. Permet de ramener des herbivores différents à un équivalent énergétique de besoins en consommation d'aliments. Le critère d'UGB/ha représente un chargement, ici en bovins, sur l'exploitation.

${ }^{5}$ Les citations sont extraites d'entretiens d'éleveurs.
}

Respecter les "savoirs " des vaches, leur faire plaisir, leur laisser de la liberté, est un moyen de favoriser la production laitière

Les éleveurs prêtent aux vaches des savoirs, sur les qualités des aliments, leurs besoins et les lieux qui leur conviennent : «Elles savent bien, si l'herbe n'est pas bonne, elles ne veulent plus y aller » (Bernard). Ils leur reconnaissent des compétences qu'eux-mêmes n'ont pas.

Ces savoirs prêtés aux vaches amènent les éleveurs à leur laisser d'importantes possibilités d'initiative : «Avec les bêtes, c'est pas vraiment nous qui commandons, on a bien nos idées, mais, entre les bonnes et les mauvaises. . . Moi, je les laisse choisir » (Denis).

Les réactions de plaisir ou de déplaisir des vaches, face à un aliment ou au lieu où elles se trouvent, et le bienêtre que leur procure une certaine liberté sont souvent évoqués dans les entretiens : " elles sont contentes d'aller, elles aiment mieux le regain » (Bernard) ou, au contraire, « elles l'aiment moins, on le voit » (Renée).

\section{Les façons de juger l'herbe pour l'alimentation des vaches}

Le critère de jugement de l'herbe, c'est « la quantité qu'elles en mangent » : "C'est la quantité que la vache mange, qui compte » (Denis). Et les vaches mangent de l'herbe en grande quantité quand elles l'aiment, quand elles manifestent des signes de plaisir en la voyant: «Elles sont contentes d'aller, elles la mangent, faut voir! » (Renée) ; « elles aiment bien ou elles aiment mieux », en opposition à : «elles veulent plus aller, elles regardent avant de manger, elles mangent pas de bon cœur, elles en mangent vraiment un peu».

À cette herbe que les vaches aiment sont associés d'autres qualificatifs, visuels : "de la bonne herbe, de l'herbe jeune, de $10 \mathrm{~cm}$, toujours verte, qui repousse». Au contraire, l' « herbe qu'elles ne mangent pas » est « haute, grande, qui monte, trop dure, trop vieille, jaune » et qualifiée de «foin ou paille». 


\section{La conception de la conduite de l'alimentation} au pâturage

Plus qu'une valeur nutritive qui serait attribuée à l'herbe, c'est le fait que les vaches l'aiment qui apparaît comme notion intermédiaire entre l'aliment et le lait : «Si tu as des artificielles où elles ne se plaisent pas, où l'herbe n'est pas bonne, le lait suit aussi, si elles n'aiment pas » (Antoine).

On a donc les principes d'alimentation suivants :

Les vaches aiment un aliment $\longrightarrow$ elles en mangent beaucoup $\longrightarrow$ elles sont contentes $\longrightarrow$ elles produisent du lait.

\section{La conception de l'herbe comme ressource}

\section{La pousse et la repousse de l'herbe}

Les éleveurs enquêtés parlent de vitesse de croissance et de transformation de l'herbe. Ils ne parlent pas de quantités d'herbe. La saison est séparée en deux périodes, et la séparation se situe quand " $l^{\prime}$ herbe devient dure», quand les vaches refusent de la manger. Avant que l'herbe ne devienne dure, les éleveurs parlent de "pousse de l'herbe ». Après, ils parlent de « repousse ».

Pour les différentes périodes de l'année, il existe des normes de croissance de l'herbe : «Là où on a la meilleure pousse, c'est au mois de juin ; au mois d'août, en général, ça repousse quand même moins vite » (Denis). Elles ne sont pas quantifiées, c'est plutôt la différence par rapport à la normale qui est notée : "La pousse ne se fait pas comme normalement »; «il y a eu une mauvaise pousse» ; «il y a encore une bonne pousse ».

À l'intérieur de ces grandes périodes, la pousse et la repousse ne sont pas évaluées de la même façon. Dans l'esprit des éleveurs, la pousse inclut la transformation de l'herbe induite par la montaison. Deux choses la caractérisent :

(i) elle est considérée sur le même plan que les événements climatiques qui l'influencent. Leurs variations sont décrites conjointement, et utilisées l'une pour l'autre. Lorsque les éleveurs élaborent des scénarios alternatifs de la forme «si a, alors b, et si c, alors d », les situations a et c se caractérisent indifféremment par une qualification de la pousse ou par une qualification du climat. Par exemple: «Si la pousse va trop vite, elles font plus de refus. Si c'est une année sèche, il n' y a pas tant à broyer » (Bernard);

(ii) elle n'est pas localisée, en ce sens que les éleveurs n'en parlent pas comme de l'évolution de l'herbe au même endroit (dans la même station) entre deux moments. Ils conçoivent l'évolution de l'herbe dans le temps, mais dans des endroits qui varient aussi selon le moment, c'est-à-dire ceux qu'ils considèrent pour $\mathrm{y}$ mettre les vaches à ce moment.
La repousse désigne à la fois la croissance de l'herbe et la ressource créée : « elles mangent la repousse ». Contrairement à la pousse, la vitesse de la repousse est peu associée au climat, mais plutôt à des opérations techniques : «il remet un peu d'engrais, et puis ça repousse » (Renée) ; «c'est important, au début, de ne pas aller trop loin [dans le fait de râper l'herbe] parce qu'il y a une meilleure repousse » (Antoine). Comme la pousse, la repousse dont parlent les éleveurs n'est pas localisée, mais ils parlent de l'évolution de l'herbe entre des endroits différents.

\section{L'effet des engrais}

Ce que les éleveurs enquêtés nomment engrais, ce sont les engrais minéraux achetés à l'extérieur de l'exploitation. Ils ont pour effet d'augmenter la vitesse de repousse. Dans la conduite du pâturage, ils sont employés après la fauche des refus. Mais ils sont rarement utilisés pendant la première partie du pâturage, à cause des risques de débordement ultérieur par l'herbe : " Finalement, le temps revient, et puis l'herbe monte comme ça, et puis on est obligé de mettre les vaches dedans » (Antoine).

\section{Les refus}

Le terme de refus est employé par les éleveurs pour désigner des touffes d'herbe mal mangées après un passage des vaches.

La quantité de refus est pour eux un moyen d'évaluer le déroulement d'un pâturage. Elle sert à comparer le pâturage de différentes exploitations, ou différentes années dans la même : «Une année où il y a trop de pousse, elles arrivent, elles laissent des refus » (Bernard).

Impérativement, assez vite dans la saison, ces refus doivent être fauchés. Surtout, il faut nettoyer les pâtures : «si vous voyez que des refus, c'est pas des pâtures, quoi!» (Denis), et «certaines fois, de faucher les refus, ça ne résout rien. $\mathrm{Si}$, c'est propre, quoi » (Paul). Cette fauche est aussi un préalable incontournable à l'apport d'engrais.

\section{La conception des lieux et du temps dans la conduite du pâturage}

\section{Une construction progressive de la dénomination des lieux}

Sur la surface de l'exploitation, il existe quatre dénominations des lieux utilisées lorsque les animaux sont dehors : les champs, les pâtures, les foins et les regains. Les champs désignent l'ensemble des surfaces de l'exploitation; c'est le dehors, et c'est aussi ainsi que les éleveurs 


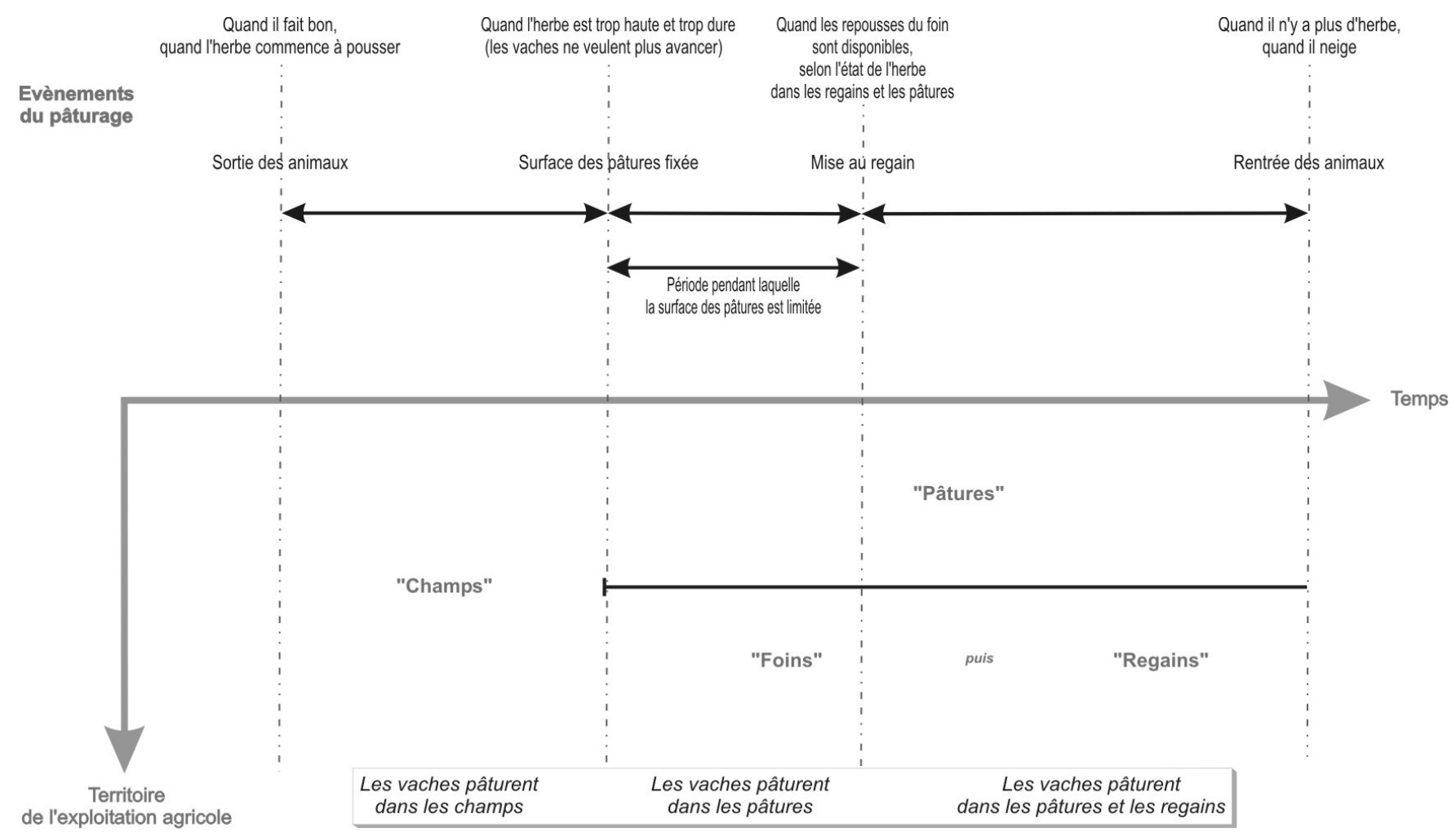

Fig. 1. Représentation de la conception de l'espace et du temps des éleveurs enquêtés au cours de la conduite du pâturage.

désignent les lieux quand sont effectuées les opérations d'entretien des prairies.

La construction des dénominations des lieux se fait progressivement au cours du déroulement de la saison de pâturage. Au début, il n'y a que des champs. Les vaches y pâturent. La dénomination de pâture n'existe que fin juin, début juillet. À ce moment, la part de l'exploitation qui sera fauchée est, par différence, celle qui sera nommée foins. Après la fauche des foins, et à partir du moment où les repousses sont utilisables, cette part est nommée regains, que ces derniers soient utilisés en pâture ou en fauche (Fig. 1).

Lorsque les éleveurs parlent de conduite de pâturage (et il n'en est évidemment pas de même dans d'autres circonstances ${ }^{6}$ ), plusieurs traits de cette façon de nommer les lieux sont à souligner. D'abord, ces lieux ne sont jamais fixés topographiquement, ou désignés par un toponyme. Ensuite, leurs noms sont aussi ceux des ressources qu'ils portent ou de la façon dont elles sont utilisées : c'est donc l'état de l'herbe qui permet de désigner le lieu. En liaison avec cette remarque, on constate que la dénomination des lieux de la conduite du pâturage n'est

\footnotetext{
${ }^{6}$ Cela ne signifie pas que la parcelle, avec son nom et des caractéristiques, n'a pas d'existence dans les conceptions des éleveurs. Mais que ce concept n'est pas utilisé lorsqu'il s'agit de la façon de concevoir la conduite du pâturage au jour le jour.
}

pas indépendante du temps. Les pâtures représentent, vers la fin de juin, ce qui a été pâturé précédemment. Cela dépend des événements pendant le printemps. Leur dénomination n'apparaît que lorsque leur totalité est déterminée.

De même, pendant la description à un niveau plus fin du déroulement du pâturage, les lieux ne sont pas nommés indépendamment du temps : ce sont les endroits où l'on se trouve en pensée, au moment évoqué par l'éleveur, dont il est question : «je dis tiens, on va laisser ça en pâture » (Denis), ou «si on manque d'herbe, on va où il faut aller » (Bernard). Ces endroits non indépendants du temps sont qualifiés soit par la description des états de l'herbe : «On ne peut pas mettre les vaches dans du foin [là où l'herbe est haute et pourrait être fauchée pour faire du foin] » (Denis), soit par des événements, antérieurs dans la saison, qui déterminent l'état de l'herbe : «Les terrains sur lesquels on a passé les génisses, on peut mettre les vaches dessus » (Renée).

\section{La conception du temps}

Les grands moments de pousse de l'herbe ou d'interventions sont exprimés au niveau du mois, quelquefois précisés à la dizaine ou à la quinzaine : « À partir du mois de juillet, à partir du 15-20 juillet, elles partent au regain » 
(Robert). Avec cette précision, les agriculteurs peuvent décrire des phénomènes qui se reproduisent tous les ans.

Mais dès que les événements sont plus ponctuels, toute référence à une date ou à une durée calendaire est récusée. À la question de l'agronome : «Quand est-ce que vous partagez [les pâtures]? », un éleveur répond : «Bah, ça dépend de l'époque, ça dépend tout du temps, ça dépend comment l'herbe poussera. Y a des années, $c^{\prime}$ est plus en avance que d'autres. On peut pas vraiment fixer de date » (Bernard).

Effectivement, toute recherche d'une datation précise d'un événement se heurte à l'implacable : "ça dépend $\mathrm{du}$ temps ». Les agriculteurs ne parlent pas en date ou en durée; les mots qu'ils emploient sont «moment » et «période».

Ces derniers sont alors rapportés à des événements climatiques ou à des états de l'herbe : "On essaie de commencer les foins le plus tôt possible, avant fin mai. Après, on a souvent une période de flotte» (Antoine). $\mathrm{Ou}$ : «Il y a un moment, les pâtures, faut les nettoyer. $C^{\prime}$ est quand il y a des refus » (Denis).

\section{La définition de l'herbe-moment-endroit}

Quand les éleveurs enquêtés parlent de la conduite du pâturage, ils ne séparent pas le moment auquel ils font référence, l'endroit auquel ils pensent, associé à ce moment, et l'état de l'herbe à cet endroit et à ce moment. Je définis la notion $\mathrm{d}^{\prime}$ « herbe-moment-endroit ${ }^{7} »$. C'est ce qu'utilisent ces éleveurs pour concevoir la conduite du pâturage dans l'espace-temps. Les vaches vont d'herbeendroit en herbe-endroit, et c'est sur des herbe-endroits que les éleveurs prévoient les évolutions de l'herbe dans un futur proche.

On a à la fois comme principe de construction et comme conséquence de cette notion les assertions suivantes concernant la conception de l'espace-temps par les éleveurs enquêtés :

(i) la pousse et la repousse de l'herbe sont appréciées par les éleveurs dans des endroits différents selon les différents moments ;

(ii) les lieux évoqués lorsqu'ils parlent de la conduite du pâturage sont nommés par des mots différents selon la période, et ces mots renvoient à l'état de l'herbe ou à son utilisation ;

(iii) les moments évoqués lorsqu'ils parlent de la conduite du pâturage sont repérés par des états d'herbe.

\footnotetext{
${ }^{7}$ Le terme d' « endroit» a été choisi en liaison avec le fait que les éleveurs ne spécifient pas de localisation pour les endroits dont ils parlent.
}

\section{Les indicateurs de la conduite du pâturage}

\section{C'est sur du temps et des vitesses que les éleveurs \\ s'appuient pour raisonner la conduite, et non pas sur des surfaces}

\section{- Le temps dont on dispose, et non la surface disponible}

Un élément essentiel dans l'esprit des éleveurs pour la conduite du pâturage est constitué par les marges laissées par le temps:

(i) avant que n'apparaisse la dénomination "pâtures » d'une partie du territoire, c'est le temps dont ils disposent avant que l'herbe ne devienne dure : «Quand l'herbe pousse assez vite, on n'arrive pas à tout manger. Les années où il fait froid, l'herbe ne pousse pas beaucoup, on a plus le temps de pâturer » (Robert) ;

(ii) après qu'est apparue la dénomination "pâtures » d'une partie du territoire, c'est le temps qu'il faut pour la repousse des regains : «Il y a un moment, il faut rationner un peu, le temps qu'on fasse les foins. Ça nous permet de gagner des jours, que ça repousse ailleurs » (Bernard).

\section{- Le rationnement et la vitesse d'avancement des vaches}

La vitesse d'avancement des vaches est liée au rationnement à la clôture électrique : «Elles ont une ration, on leur donne tant par jour, on leur met un fil devant, elles ont tant à manger d'herbe » (Robert). Avec l'avancée de la clôture, chaque jour, les vaches ont à disposition, et consomment, un «herbe-moment-endroit». Le principe général du rationnement est le suivant : l'éleveur regarde ce que les vaches ont laissé le jour précédent, puis ajuste la quantité donnée en conséquence, « d'après ce qu'elles ont mangé le jour d'avant. Si il reste trop d'herbe, une fois qu'elles ont pâturé la journée, il faut leur en donner un peu moins. Si je vois que c'est bien mangé, je continue à peu près à donner ce qui peut être mangé » (Bernard). Avec ce principe général, c'est l'état de l'herbe et la réaction des vaches qui déterminent le rationnement et, en conséquence, la vitesse d'avancement.

\section{- La vitesse de pousse et d'évolution de l'herbe}

Comme nous l'avons vu, c'est sous la forme d'une vitesse de croissance et de transformation que les éleveurs enquêtés apprécient l'herbe : la pousse ou la repousse.

\section{La conduite du pâturage se fait par la confrontation de la vitesse d'avancement des vaches et de celle de la pousse ou repousse de l'herbe}

- Avant que n'apparaisse la dénomination «pâtures » d'une partie du territoire

La vitesse d'avancement des vaches est confrontée à la vitesse de transformation de l'herbe pendant la 
montaison. Ces deux vitesses ne sont pas indépendantes: quand l'herbe monte, la vitesse d'avancement est ralentie : «Si l'herbe a monté de moitié, vous avancez deux fois moins vite» (Denis). Il vient un moment où «l'herbe va plus vite que les vaches la mangent »(Bernard). En continuant à avancer, les vaches sont placées chaque jour devant des endroits où l'herbe est de plus en plus mûre et dure. Jusqu'au moment où le comportement des vaches est tel que les éleveurs ne continuent plus à les faire avancer : "elles veulent plus aller» (Renée). C'est à ce moment-là que les champs sont partagés entre «pâtures» et «foins". La partie déjà pâturée est nommée "pâtures", elle fera l'objet d'un nouveau passage des vaches; le reste est nommé «foins ». La proportion entre ces deux parties n'est pas un objet de détermination ou de prévision dans la conduite du pâturage. C'est une conséquence du temps pendant lequel les éleveurs ont pu faire avancer les vaches avant que l'herbe ne devienne dure : «On avait prévu que ce champ là, c'était pour les vaches, et puis on n'en mange que la moitié, c'est ça qui arrive » (Renée). Et ce temps dépend du climat qui fait pousser l'herbe plus ou moins vite; il ne peut donc pas être maîtrisé par les éleveurs. Ces derniers ne considèrent pas qu'il y a une concurrence entre une surface de pâture et une surface de foin : l'avancement des vaches se fait, puis, selon les circonstances, les deux surfaces respectives sont fixées : "Vers la fin juin, je commence à regarder, je dis tiens, on va laisser ça en pâture, et puis voilà. On commencera à foiner à partir de cet endroit » (Denis). Pendant toute cette période, la surface qu'il est possible de pâturer n'apparaît pas limitée.

- Après qu'est apparue la dénomination «pâtures » d'une partie du territoire, et jusqu'à la disponibilité des repousses

C'est la période pendant laquelle les éleveurs pensent que la surface possible à pâturer est limitée à celle des pâtures. Cette surface ne sera agrandie que sur les repousses après la fauche $\mathrm{du}$ foin, les regains. C'est tout l'enjeu de la conduite du pâturage dans cette région. Il s'agit d'avoir une surface de pâtures suffisante pour permettre une alimentation correcte des vaches avant la disponibilité des regains : "On a toujours peur de manquer, on a toujours peur de manquer d'herbe pour les vaches, quoi, dans les pâtures » (Renée). Et en effet, il arrive qu'« on n'a[it] pas de regain dans les champs de foin. Et puis, dans nos pâtures, on arrive au bout » (Paul). Il n'y a alors pas d'autre solution que de rationner les vaches.

À partir du moment où les repousses de regains sont suffisantes, la surface n'est plus objet de réflexion de la part des éleveurs; ils la considèrent à nouveau comme illimitée.

\section{Ces conceptions d'éleveurs face à des modèles d'agronomes}

\section{La conception de l'espace-temps}

À partir de la figure 1, rappelons que, dans les conceptions des éleveurs enquêtés :

(i) les interventions sont calées sur des états d'herbe en interaction avec le comportement des vaches;

(ii) l'espace est conçu en herbe-endroits, non fixes dans le temps. C'est l'état de l'herbe qui définit, à un moment donné, ce qu'il est possible de faire dans ces endroits.

L'outil généralement utilisé par les agronomes pour enregistrer les événements du pâturage donne une représentation différente de l'espace-temps (Fig. 2) :

(i) le temps est repéré par des jours calendaires;

(ii) l'espace est divisé en parcelles, fixes tout au long de la période de pâturage.

Ce qui est considéré là, c'est que l'herbe, dans un endroit fixe, évolue quand le temps s'écoule. Au contraire, pour les éleveurs, temps et endroits bougent ensemble, définis par l'état de l'herbe ou les actions à réaliser.

Ces différences de conceptions ont les conséquences suivantes :

(i) il est difficile aux éleveurs de faire une prévision de pâturage a priori, et de la caler sur des repères calendaires. Pour eux, les décisions sont prises en fonction de ce qu'ils voient sur le terrain à un moment donné, les herbe-endroits. Ils ne font pas de prévision sur les endroits qui seront pâturés ;

(ii) ce qui est donné chaque jour aux animaux est conçu différemment : les éleveurs considèrent qu'ils donnent une ration d'herbe; les agronomes parlent d'une surface mise à disposition des animaux ;

(iii) il est difficile aux éleveurs enquêtés de concevoir que les animaux reviennent au même endroit à des moments différents au cours de la saison. Pour eux, les herbe-endroits ne sont alors pas les mêmes, parce que l'herbe a changé.

\section{Les différences de conception des conduites de pâturage par les éleveurs enquêtés et par les agronomes dans le pâturage tournant}

L'une des difficultés de la conduite du pâturage est constituée par l'épiaison de l'herbe au printemps (mijuin sur le deuxième plateau du Jura), car les tiges formées sont de qualité médiocre, voire trop dures pour être consommées au pâturage par les vaches. Il y a deux cas pour l'herbe à cette période : soit elle est exploitée avant l'apparition des épis ou leur durcissement, et donne une repousse feuillue; soit elle n'est pas exploitée avant, 


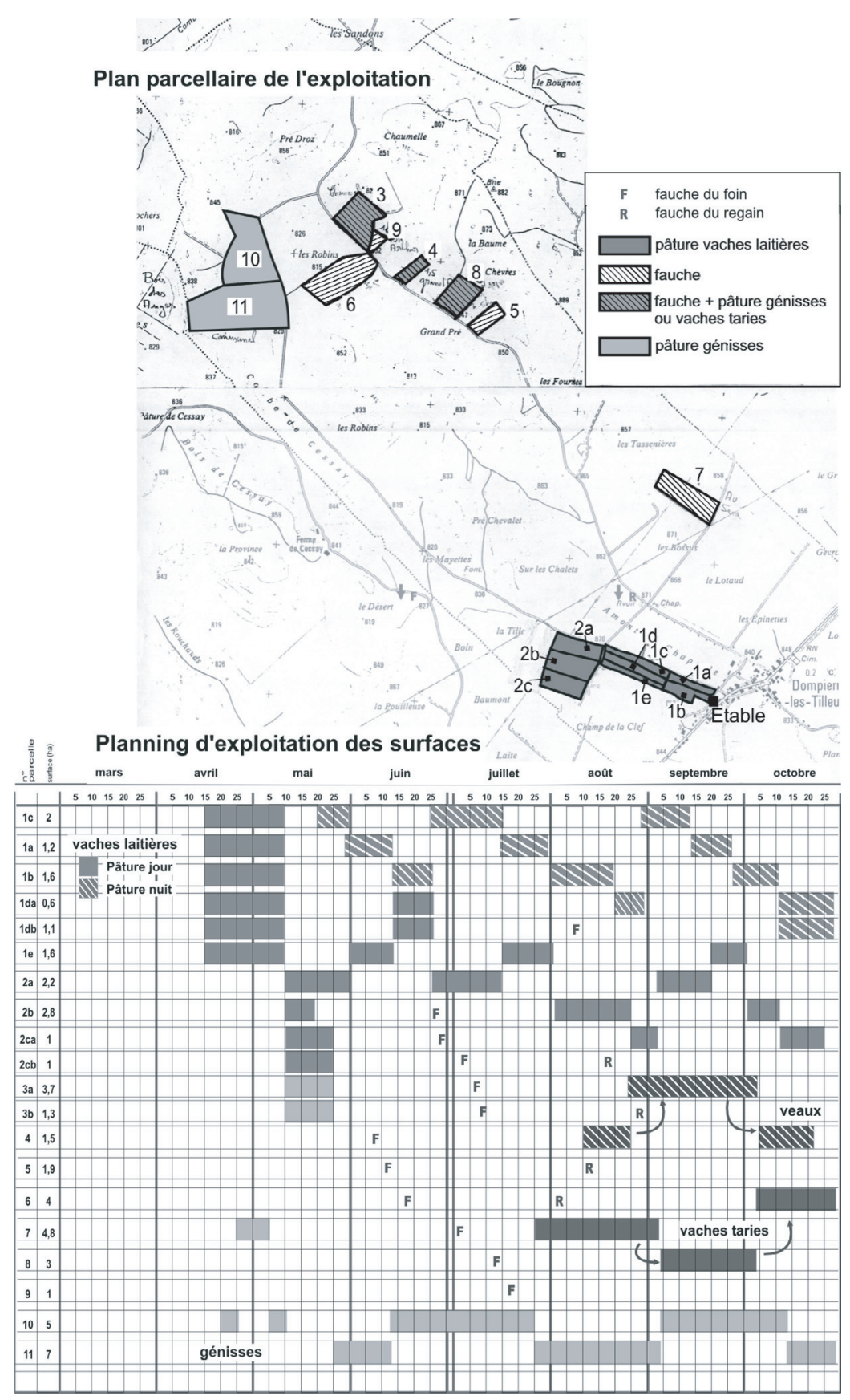

Fig. 2. Représentation d'un calendrier d'exploitation des surfaces fourragères d'une exploitation pendant une saison de pâturage, avec un plan des parcelles.

et l'herbe épiée ne peut plus être pâturée à partir d'un certain moment, elle est récoltée en foin.

La façon de prendre en compte ce phénomène est différente chez les éleveurs enquêtés et chez les agronomes qui raisonnement en terme de pâturage tournant.

Dans le premier cas, les vaches avancent en consommant chaque jour une ration, sur une surface qui paraît illimitée. Quand l'herbe épie et durcit, il vient un moment où sa consommation n'est plus possible par les vaches («elles veulent plus y aller»). Les vaches sont ramenées sur la surface déjà exploitée en pâturage, qui est alors fixée comme conséquence de ce qui s'est passé avant. Cette surface prend à ce moment le nom de pâtures. L'enjeu est qu'elle ne soit pas trop petite.

Dans le pâturage tournant, la surface qui est allouée au pâturage est déterminée a priori, avant la sortie des animaux (Lebrun, 1983). L'enjeu principal de la conduite est d'empêcher l'épiaison sur cette surface (Cristofini et Jeannin, 1979). Les vaches la parcourent plusieurs fois, de façon à ce qu'elles consomment les épis en train de pousser. Dans ce cas, les indicateurs proposés pour la conduite s'appuient sur un raisonnement en termes de « trésorerie 
fourragère » (Duru et al., 1988). Sur la surface allouée au pâturage, on calcule à partir de mesures de hauteur $\mathrm{d}$ 'herbe à un moment le volume d'herbe disponible, que l'on divise par le nombre de vaches. En relation avec des références, cette valeur permet de prendre des décisions, comme par exemple la date de début du pâturage ou le retrait d'une parcelle allouée s'il y a trop d'herbe (Duru, 2000 ; Thébaut et al., 1998). Les éleveurs enquêtés ne raisonnent pas sur cette quantité totale d'herbe puisqu'ils ne conçoivent pas cette surface allouée limitée au printemps. Par contre, cet indicateur pourrait leur rendre des services pendant la période où ils considèrent que la surface de leurs pâtures est limitée, en juillet.

Pour conduire le pâturage au printemps, les éleveurs observent surtout l'herbe qui se trouve en avant des vaches, dans des endroits où elles pourront être dans quelques jours. Quand cette herbe commence à pousser plus vite, ils essaient, dans la mesure du possible, d'augmenter la vitesse d'avancement des vaches. Mais cette dernière décision ne se prend que si l'avancement plus rapide des vaches n'a pas pour conséquence de produire derrière elles un couvert végétal de structure trop hétérogène, avec des touffes qui pourront par la suite donner lieu à des refus. Les éleveurs regardent donc aussi l'herbe qui se trouve derrière les vaches, dans des endroits où elles viennent de passer. Cela renvoie à l'indicateur de hauteur d'herbe moyenne résiduelle proposé par Duru (2000) pour la conduite du pâturage tournant. Cependant, les éleveurs repèrent plutôt des hétérogénéités du couvert qu'une hauteur d'herbe moyenne.

Les deux différences principales dans la prise en compte de l'herbe dans les conceptions de la conduite du pâturage entre agronomes et éleveurs sont les suivantes :

(i) les agronomes raisonnent sur des hauteurs d'herbe moyennes sur une surface allouée a priori au pâturage pour la période de printemps; les éleveurs regardent l'état de l'herbe dans les endroits où les vaches seront dans quelques jours ;

(ii) les agronomes et les éleveurs évaluent l'herbe derrière le passage des vaches, mais avec des indicateurs différents. Les premiers utilisent la hauteur d'herbe moyenne résiduelle dans la parcelle d'où les vaches viennent de sortir. Les seconds regardent surtout l'hétérogénéité du couvert dans les endroits où les vaches viennent de passer.

\section{La conception de l'incertitude et la gestion de l'aléa climatique}

Dans la conception des agriculteurs enquêtés, le risque, lors de la conduite du pâturage, s'exprime en termes de régularité. Il faut éviter de manquer d'herbe à un moment donné, donc disposer d'un herbe-endroit dans un état convenable à chaque moment. Au printemps, comme à partir d'août, les agriculteurs considèrent que la surface à pâturer n'est pas limitée. Disposer d'un herbe-endroit chaque jour ne leur pose pas question; «quand il n'y a plus d'herbe, on va plus loin» (Bernard). C'est en juillet, pendant la période où les pâtures sont limitées et la repousse lente, que le risque de manquer existe. Cette surface de pâtures, on l'a vu, dépend de la durée avant que l'herbe ne devienne dure, qui elle-même dépend de la pousse de l'herbe, donc du climat. Avec les conduites habituelles du pâturage de la région, il n'y a pas de moyens de maîtrise de la conduite avant juillet, ni beaucoup de possibilités d'action : quand la date de sortie des vaches est déterminée, il n'y a plus qu'à suivre l'herbe qui pousse. D'où peut-être une expression exprimant la peur, et non le risque. Les êtres vivants en cause ne sont pas forcément maîtrisables : «l'herbe va plus vite que les vaches la mangent » est une phrase de description du fonctionnement du pâturage qui ne laisse pas beaucoup d'initiative possible dans la conduite. Dans cette conception, la pousse de l'herbe est fixée à l'extérieur, et l'enjeu est la maîtrise de la surface des pâtures en juillet. Un nouveau mode de conduite qui est une innovation locale est mis en place par certains agriculteurs, avec une sortie des vaches très précoce et un pâturage très ras au début de la saison. La pousse en juin est alors ralentie, et cela permet aux éleveurs de faire avancer plus vite leurs vaches à cette période. Cela permet d'avoir une meilleure maîtrise de la surface des pâtures en juillet.

Dans la conduite du pâturage tournant telle qu'elle est conçue par les agronomes, nous avons vu que la surface enjeu de la conduite du pâturage est la surface du printemps. Il s'agit que les vaches, en passant plusieurs fois sur les pâtures au printemps, coupent les tiges qui montent avant que les épis ne se forment et ne durcissent. L'herbe est alors toujours feuillue et de bonne qualité pour les vaches. C'est, à partir des calculs de trésorerie fourragère, en modifiant la surface à chaque passage que se font les ajustements en fonction de la pousse de l'herbe. Les moyens de s'ajuster aux variations du climat et d'acquérir une nouvelle maîtrise ne sont donc pas les mêmes que pour les agriculteurs.

Par ailleurs, lorsque l'objectif assigné à l'aide à la conduite du pâturage par les agronomes consiste à ajuster l'herbe produite à l'herbe consommée, pour dimensionner au mieux les surfaces utilisées et l'azote apporté, cela introduit évidemment une majoration de la problématique du risque : le fait d'accepter d'avoir trop d'herbe peut constituer une régulation qu'utilisent en effet les éleveurs enquêtés. Ils posent la question de la conduite des refus en juillet, plutôt que celle de diminuer leur occurrence par une conduite au printemps. Ainsi, Antoine dit : «Je ne fauche pas les refus, on ne sait jamais, on peut en avoir besoin. J'en fauche toujours quand même 
un petit peu, mais pas tout ce que je devrais, quoi... » Cette conduite des refus n'est pas facile à raisonner pour les agriculteurs enquêtés, d'autant qu'elle intervient pendant la période délicate de juillet. Vaut-il mieux alors faucher pour obtenir une repousse "comme du regain", mais avec huit jours de retard, ou laisser l'herbe repousser entre les refus et perdre à cause d'eux de la surface à pâturer par les animaux ? La constitution de références sur ces questions pourrait leur être une aide.

\section{Discussion - conclusion}

Les tâches des agronomes consistent à proposer de nouveaux systèmes de pâturage, ou des indicateurs pour évaluer ceux qui sont déjà en œuvre. Des outils utilisés actuellement sont des simulateurs de pâturage. On peut s'interroger sur l'utilisation qui peut être faite dans ces modèles des connaissances sur les conceptions des agriculteurs.

En fait, ces connaissances sur les conceptions des agriculteurs d'une localité sur leur conduite de pâturage prennent de l'intérêt dès lors que les agronomes se posent la question de la transformation de ces conduites dans les exploitations. Cela permet d'expliquer certaines des difficultés de ces agriculteurs à comprendre des prescriptions, en particulier pour la conduite du pâturage tournant. La différence dans la façon de prendre en compte la surface en est un exemple. Deux conséquences peuvent être envisagées.

D'abord, la proposition d'indicateurs pour le pilotage du pâturage doit être accompagnée de projets de formations pour éventuellement modifier les conceptions des agriculteurs locaux qui souhaitent mettre en place un pâturage tournant. Mais, par ailleurs, la connaissance des conceptions de ces agriculteurs peut amener les agronomes à s'interroger sur leurs propres conceptions. Ainsi, le fait de penser en termes de surface de pâturage dès le printemps conduit les agronomes à se poser des questions difficiles sur les dimensionnements de cette surface, et ses ajustements en cours de conduite, comme dans Coleno (1999). Gillet et al. (1980) remarquent les difficultés de raisonnement introduites par le fait de considérer des surfaces pâturées fixes. Avec cette notion, le temps de repos entre deux passages des animaux au même endroit est égal au temps que les animaux ont mis pour parcourir le reste de la prairie. Cela porte ces auteurs à conclure que «cette liaison entre le temps de passage et le temps de repos est une contrainte supplémentaire qui rend encore plus difficile toute planification. Pour l'éviter, il faudrait que la surface parcourue par les animaux puisse être modifiée à volonté d'un passage à l'autre. Mais une prairie a une surface fixe ». Ce qui est vrai. Cependant, les agriculteurs, ne faisant pas de partition au départ entre foin et pâture, raisonnent comme si la surface disponible pour le pâturage ne l'était pas. D'autre part, une réflexion pourrait avoir lieu sur la prise en compte d'indicateurs de vitesses, d'avancement des vaches et de croissance $\mathrm{d}$ 'herbe, à cette période.

Des simulateurs de pâturage sont actuellement utilisés pour évaluer des stratégies de pâturage dans une région (Cros et al., 2001), ou pour accompagner la conduite (Delaby et al., 2001). Ces simulateurs comprennent deux parties : un système biotechnique qui modélise les réactions de l'herbe, et un système de décision qui représente une conduite formalisée en règles de décisions, liées à l'état d'indicateurs. On peut penser qu'il serait intéressant d'y introduire des règles et des indicateurs provenant des conceptions des éleveurs.

La généralisation des résultats obtenus ici pose deux questions. Il est évident qu'ils ne peuvent intéresser les agronomes que s'ils dépassent le niveau d'un village jurassien. Nous verrons que quelques éléments de bibliographie les confortent en partie. Mais il est possible aussi d'envisager qu'il y ait des systèmes de pensée localisés qui soient particuliers et nécessitent une adaptation des prescriptions ou des aides à la décision.

L'analyse d'un entretien en Lorraine montre que la façon de concevoir sa conduite du pâturage est la même pour cet éleveur que pour ceux que j'ai étudiés ici (Fiorelli, 2004; Mathieu et Fiorelli, 2002). Meuret (1993), pour des troupeaux ovins gardés, montre l'importance du comportement des animaux et de la relation au troupeau pour les actions du berger. Cet auteur souligne que, au niveau du pilotage journalier du pâturage, espace et temps sont très liés pour les bergers. En élaborant une ration journalière, ils conduisent leurs troupeaux à des ressources-moments-endroits divers dans la journée, mais réguliers et prévisibles pour le troupeau. Landais et Deffontaines (1988) étudient les pratiques d'un berger qui garde des moutons dans un alpage. Là encore, le comportement du troupeau est un indicateur essentiel de la conduite du pâturage. Et le berger cherche aussi à construire une ration journalière en considérant les états d'herbe d'endroit en endroit. Plus généralement, Friedberg et al. (2000) montrent, à propos des pratiques pastorales sur le causse Méjan, qu'il existe des décalages entre les façons qu'ont les éleveurs de concevoir l'embroussaillement et les mesures qui leur sont proposées pour restaurer une ouverture des prairies. Plus loin, et à un niveau de temps plus long, Figuié (2001) étudie les représentations de la dégradation de leurs pâtures par des agriculteurs brésiliens. L'auteur souligne la vision dynamique que ces agriculteurs ont de la dégradation. Ils raisonnent sur des vitesses de transformation, par rapport à des diagnostics réalisés sur l'état du couvert végétal. Les comportements d'animaux (des bovins) servent aussi à définir la dégradation.

Remarquons que ces études ont été réalisées dans des conditions plutôt extensives. Pour notre propos sur 
la conduite du pâturage, il serait intéressant d'étendre l'analyse des conceptions d'éleveurs sur la conduite du pâturage à des zones plus intensives, comme la Bretagne, là où le pâturage tournant semble être une pratique plus généralisée (Grasset et al., 1997).

Le niveau étudié ici est celui de la mise en œuvre de la conduite au cours de la saison. C'est la façon dont des agriculteurs conçoivent ce que l'on peut appeler la tactique, ou le pilotage. Bellon et al. (1999) proposent, au travers des saisons-pratiques, une représentation de la conduite des surfaces et des troupeaux à un niveau plus global, nommé stratégie, pour l'ensemble de l'exploitation et toute l'année. Ces auteurs constatent des régularités dans les affectations de parcelles. Une analyse du système de pensée des éleveurs du Jura sur ce niveau me semblerait intéressante de façon à interpréter la conduite au jour le jour dans le cadre plus général des options stratégiques de l'exploitant. Toutefois, la démarche d'étude pour rendre celles-ci intelligibles reste encore à préciser, car elle n'est vraisemblablement pas identique à celle qui a été utilisée ici, même si elle peut reposer sur les mêmes principes théoriques.

La démarche mise en œuvre pour établir ces résultats est une démarche pluridisciplinaire, entre l'agronomie et la socio-anthropologie. Elle témoigne d'un "passage de frontières » (Jollivet, 1992). Ce passage, ou plutôt ces passages se sont réalisés dans des conditions particulières de pluridisciplinarité : c'est l'agronome elle-même qui a mis en évidence les conceptions des agriculteurs sur le processus technique qui est son objet d'étude (Darré et al., 2004). Il ne s'agit pas seulement de s'approprier une nouvelle méthode. En passant des sciences de l'ingénieur aux sciences sociales, il faut opérer un véritable changement de posture par rapport aux acteurs et à leurs pratiques.

\section{Références}

Aubry, C., 2000. Une modélisation de la gestion de production dans l'exploitation agricole, Revue française de gestion, 129, 32-46.

Bellon, S., Girard, N., Guérin, G., 1999. Caractériser les saisonspratiques pour comprendre l'organisation d'une campagne de pâturage, Fourrages, 158, 115-132.

Coléno, F.C., 1999. Le pâturage des troupeaux laitiers en question : contribution d'une analyse des décisions des éleveurs, Fourrages, 157, 63-76.

Critofini, B., Jeannin, B., 1979. Quelques réflexions sur l'intensification du pâturage raisonné en système. Un exemple dans les conditions du Plateau Lorrain, Motorisation et techniques agricoles, novembre, 1-3.

Cros, M.-J., Duru, M., Peyre, D., 2001. SEPATOU : simulation de conduites de pâturage-exemple des «menus » bretons, Actes des Journées de l'AFPF, 21-22/03/2001, 153-165.

Darré, J.-P., 1985. La Parole et la technique. L'univers de pensée des agriculteurs du Ternois, Paris, L'Harmattan.
Darré, J.-P., Lasseur, J., Landais, E., Hubert, B., 1993. Raisons et pratiques. Dialogue avec un éleveur ovin, Études rurales, 131-132 ; Paris, EHESS, 107-180.

Darré, J.-P., Mathieu, A., Lasseur, J. (Eds), 2004. Le Sens des pratiques : conceptions d'agriculteurs et modèles d'agronomes, Paris, INRA Éditions.

Delaby, L., Peyraud, J.-L., Faverdin, P., 2001. Pâtur'IN : le pâturage des vaches laitières assisté par ordinateur, Actes des Journées de l'AFPF, 21-22/03/2001, 167-177.

Duru, M., Fiorelli, J.-L., Osty P.-L., 1988. Propositions pour le choix et la maîtrise du système fourrager. I. Notion de trésorerie fourragère, Fourrages, 113, 37-56.

Duru, M., 2000. Le volume d'herbe disponible par vache : un indicateur synthétique pour évaluer et conduire un pâturage tournant, INRA Prod. Anim., 13, 5, 325-336.

Figuié, M., 2001. La Construction sociale d'un savoir sur la dégradation des ressources naturelles: le cas des pâturages dans les exploitations agricoles familiales de la commune de Silvânia au Brésil. Thèse, INA P-G.

Fiorelli, J.-L., 2004. Petits arrangements avec le fil. De l'herbe au calendrier de pâturage dans les Vosges, in Darré, J.-P., Mathieu, A., Lasseur, J. (Eds), Le Sens des pratiques : conceptions d'agriculteurs et modèles d'agronomes, Paris, INRA Éditions, 147-156.

Friedberg, C., 1997. Diversité, ordre et unité du vivant dans les savoirs populaires, Natures Sciences Sociétés, 5, 1, 5-17.

Friedberg, C., Cohen, M., Mathieu, N., 2000. Faut-il qu'un paysage soit ouvert ou fermé? L'exemple de la pelouse sèche du causse Méjan, Natures Sciences Sociétés, 8, 4, 26-42.

Gillet, M., Huguet, L., Breisch, H., Le Roy, A., Posny, P., 1980. Peut-on concilier pâture et plein emploi des graminées fourragères? Nouvelle approche d'un vieux problème. II. Problème d'exploitation des graminées en pâturage rationné : les conditions d'un système de pâturage, Fourrages, 81, 3-20.

Girard, N., 1995. Modéliser une représentation d'experts dans le champ de la gestion de l'exploitation agricole. Stratégies d'alimentation au pâturage des troupeaux ovins allaitants en région méditerranéenne. Thèse de doctorat en biométrie, Université Claude Bernard - Lyon I.

Grasset, M., Roger, P., Dequin, A., Follet, D., Thebault, M., Berkani M.E., Le Gall, A., 1997. Étude des systèmes fourragers laitiers maximisant le pâturage en Bretagne : synthèse des résultats 95 et 96, analyse du fonctionnement et mise au point d'indicateurs, Renc. Rech. Ruminants, 4, 9-14.

Jollivet, M. (Ed.), 1992. Sciences de la nature, sciences de la société. Les passeurs de frontière, Paris, CNRS Éditions.

Landais, E., Deffontaines, J.-P., 1988. André L. : un berger parle de ses pratiques (Document de travail, Inra SAD Versailles, Dijon, Mirecourt), Versailles, INRA.

Landais, E., Deffontaines, J.-P., 1990. Les pratiques des agriculteurs. Point de vue sur un courant nouveau de la recherche agronomique, in Brossier, J., Vissac, B., Le Moigne, J.-L. (Eds), Modélisation systémique et système agraire, Actes du séminaire du département de recherche sur les Systèmes agraires et le développement, Saint-Maximin, mars 1989, 31-64.

Lebrun, V., 1983. Comment gérer le pâturage? Paris, Document de l'Institut technique de l'élevage bovin. 
Mathieu, A., 2004. Parcelles et endroits d'éleveurs : la conduite du pâturage dans le Jura, in Darré, J.-P., Mathieu, A., Lasseur, J. (Eds), Le Sens des pratiques : conceptions d'agriculteurs et modèles d'agronomes, Paris, INRA Éditions, 157-173.

Mathieu, A., Fiorelli, J.-L., 1990. Modélisation des pratiques de pâturages d'éleveurs laitiers dans le Nord-Est. Les régulations face à l'aléa climatique, in Brossier, J., Vissac, B., Le Moigne, J.-L. (Eds), Modélisation systémique et système agraire, Actes du séminaire du département de recherche sur les Systèmes agraires et le développement, Saint-Maximin, mars 1989, 135-157.

Mathieu, A., Fiorelli, J.-L., 2002. Some difficulties in rotational grazing management linked to farmer's conception of time and space, in Multi-function grasslands, Proceedings of the 19th general meeting of the European Grassland Federation, La Rochelle, France, 27-30 May 2002, 1102-1103.

Reçu le 2 décembre 2003. Accepté le 2 septembre 2004.
Meuret, M., 1993. Piloter l'ingestion au pâturage, in Landais, E. (Ed.), Pratiques d'élevage extensif, identifier, modéliser, évaluer, INRA Éditions, 161-198.

Moisan, H., 1988. Développement agricole et localités. Chemin critique d'une pratique de recherche sociologique dans les territoires villageois de la Plaine Ouest des Vosges. Thèse, Université Paris X - Nanterre.

Papy, F., 1994. Working knowledge concerning technical systems and decision support, in Dent, J.B., Mc Gregor, M.J. (Eds), Rural and farming systems Analysis, European perspectives, CAB International, 222-235.

Prieto, L.J. 1975. Pertinence et pratique, Paris, Éditions de Minuit. Thébaut, M., Dequin, A., Follet, D., Grasset, M., Roger, P., 1998. Dossier 5 menus pour vaches laitières au pâturage : le pâturage au quotidien, du plan d'alimentation à la conduite de l'herbe. Guide pratique de l'éleveur. Chambre d'agriculture - EDE de Bretagne, Institut de l'élevage.

To access this journal online: www.edpsciences.org 